\title{
Additional TWA members?
}

\section{Spectroscopic verification of kinematically selected TWA candidates}

\author{
I. Song ${ }^{1}$, M. S. Bessell ${ }^{2}$, and B. Zuckerman ${ }^{1}$ \\ 1 Department of Physics and Astronomy (UCLA), Los Angeles, CA 90095-1562, USA \\ 2 Research School of Astronomy and Astrophysics, The Australian National University, ACT 2611, Australia
}

Received 27 September 2001 / Accepted 24 January 2002

\begin{abstract}
We present spectroscopic measurements of the 23 new candidate members of the TW Hydrae Association from Makarov \& Fabricius (2001). Based on $\mathrm{H} \alpha$ and $\mathrm{Li} 6708 \AA$ strengths together with location on a colormagnitude diagram for Hipparcos TWA candidates, we found only three possible new members (TYC 7760-08351, TYC 8238-1462-1, and TYC 8234-2856-1) in addition to the already known member, TWA 19. This eliminated most of the candidates more distant than 100 pc. Three Tycho stars, almost certainly members of the Lower Centaurus Crux association, are the most distant members of the TWA. A claim of isotropic expansion of TWA has to be re-evaluated based on our new results. Generally, one cannot identify new members of a diffuse nearby stellar group based solely on kinematic data. To eliminate interlopers with similar kinematics, spectroscopic verification is essential.
\end{abstract}

Key words. galaxy: open clusters and associations: individual: TWA - stars: kinematics - stars: planetary systems - techniques: spectroscopic

\section{Introduction}

During the past few years, a handful of young ( $<100$ Myrs) and nearby $(\leq 100 \mathrm{pc})$ groups of stars have been identified. Usually, they occupy a few hundred square degrees in the plane of the sky (thus are less conspicuous than compact clusters) and are not associated with any known interstellar clouds. Therefore they have been hard to find and have remained unnoticed until recently. Beginning with the TW Hydrae Association ("TWA" Zuckerman et al. 2001b, and references therein), currently, there are about eight such associations reported in the literature. For the most upto-date information on these groups of stars, readers are refered to Jayawardhana \& Greene (2001).

Thermal emission from massive planets can be detectable when they are separated far enough from the primary stars. For a given linear separation between primary and planet, a closer system to Earth will provide a larger angular separation making detection of the planet easier. Planets around young stars are still hot and they emit a fair amount of infrared radiation. Therefore, young and nearby stars are excellent targets for observational searches for giant planets and dusty "proto-planetary" disks. The TWA $(d \sim 60 \mathrm{pc})$, the $\beta$ Pictoris moving group $(d \sim 35 \mathrm{pc})$, and the Tucana/HorA Association

Send offprint requests to: I. Song,

e-mail: song@astro.ucla.edu $(d \sim 45 \mathrm{pc})$ are the three youngest and closest groups to Earth; hence they form the best targets for ground- and space-based searches for planets. The Capricornus group (van den Ancker et al. 2001) is also a very young nearby group, however it is almost certainly a subgroup of the bigger $\beta$ Pictoris group (Zuckerman et al. 2001a).

There are currently 19 known star systems in TWA and most of its members have been observed with the Hubble Space Telescope and/or with a groundbased telescope with adaptive optics (AO) capability. Some TWA members possess substellar companions (TWA 5; Lowrance et al. 1999) or prominent dusty disks (TW Hydrae, HR 4796A, Hen 3-600, and HD 98800). Any newly identified members of TWA, therefore, are excellent targets for planet or planetary disk searches.

Using a modified convergent point method, Makarov \& Fabricius (2001, MF hereafter) found 23 new kinematic candidate members of TWA beside eight known members. In fact, one of the 23 candidates is a known TWA member, TWA 19 (HIP 57524; Zuckerman et al. 2001b). Based on a kinematic model of TWA including the candidate members, MF predicted radial velocities of the 23 new candidates. They also suggest that TWA expands with a rate of $0.12 \mathrm{~km} \mathrm{~s}^{-1} \cdot \mathrm{pc}^{-1}$ and that TWA is part of a larger structure, the "Gould disk", rather than a separate group of stars. Considering the importance of TWA members as aforementioned observational targets and as 
corner-stones in investigations of the surrounding environment (local association, Sco-Cen complex, etc.), all of the MF candidates need to be verified spectroscopically. In this paper, we present spectroscopic data for all MF candidates and discern true members from interlopers based on spectroscopic youth indicators.

\section{Observations}

We are in the midst of an extensive survey to search for young and nearby stars to Earth at Siding Spring Observatory (Australia) and Lick Observatory (USA). We took spectra of all $23 \mathrm{MF}$ candidates during January, April and June 2001 and January 2002 observations at SSO. We used an echelle spectrograph on the Nasmyth-B focus of the Australian National University's $2.3 \mathrm{~m}$ telescope. Eight orders of the echelle spectrum cover portions of the wavelength range from $5800 \AA$ to $7230 \AA$. We focused on orders which contained the $\mathrm{H} \alpha$ and $\mathrm{Li} 6708 \AA$ lines. In these orders, the measured resolution was $0.40 \AA$. Radial velocities were determined by cross-correlating target and radial velocity standard spectra over 5 or 6 orders of the echelle which were chosen to produce strong correlations and have few atmospheric features. Projected rotational velocities were measured from a total of about 10 lines in the Li $6708 \AA$ echelle order with a procedure similar to that of Strassmeier et al. (1990). Equivalent widths of $\mathrm{H} \alpha$ and $\mathrm{Li} 6708 \AA$ were measured using the IRAF task splot.

Torres et al. (2001) measured radial velocities of 9 stars, four (TWA 5, TYC 6604-0118-1, HIP 48273 and HIP 50796) of which we also observed. Three of the common stars (TWA 5, TYC 6604-0118-1, and HIP 48273) are known binaries for which Torres et al. (2001) extracted binary orbits. The binarity can explain discrepancies (Table 1 ) between our measured radial velocities and those of Torres et al. (2001). Our measured radial velocity of HIP 50796 differs from that of Torres et al. (2001) by $\sim 10 \mathrm{~km} \mathrm{~s}^{-1}$. On the night of our HIP 50796 observation, the extracted radial velocities of two radial velocity standard stars were consistent with those from published catalogs within the errors $\left(\sim 2 \mathrm{~km} \mathrm{~s}^{-1}\right)$. Thus, we believe that our measurement is correct and HIP 50796 is likely a spectroscopic binary also. Later HIP 50796 turned out to be a non-TWA star. A list of 31 stars from MF with our spectroscopic data are summarized in Table 1.

\section{Results}

Because of the high fraction of binaries among young stars (see Patience et al. 1998, for example), oftentimes, stellar radial velocities are affected by binary orbital motions. Therefore, we cannot simply compare MF's predicted radial velocities with measured ones to accept or reject the candidates. In addition, MF assumed an isotropic expansion of TWA. If the assumption is not correct, comparing predicted and measured radial velocities is meaningless.

The very young age of TWA ( $\leq 10$ Myrs) enables us to select true TWA members based on their strong Li $6708 \AA$ absorption line strength depending on the spectral type of the candidates. By comparing equivalent widths of Li $6708 \AA$ line for all candidates with those of comparably young open clusters members (Fig. 1), we can eliminate more than half of the candidates. The eliminated candidates have too weak a $\operatorname{Li} 6708 \AA$ absorption line to be a $\leq 10$ Myr old star.

Generally, if a late K- or early M-type star that is not an interacting binary shows $\mathrm{H} \alpha$ in emission or partially filled-in, then the star is young. However, even very young stars such as TWA members, if earlier than mid-K, usually do not show $\mathrm{H} \alpha$ in emission. Most of the MF candidates are earlier than mid-K, thus, we have not used $\mathrm{H} \alpha$ as a tool to reject candidates.

The age of TWA ( 10 Myrs $)$ falls between those of NGC 2264 (<10 Myrs) and IC 2602 (10 30 Myrs). Equivalent widths of previously known TWA members (symbol "T" in Fig. 1) nicely fall between the regions encompassing NGC 2264 and IC 2602. Therefore, we can use the lower envelope of IC $2602 \mathrm{Li} 6708 \AA$ equivalent widths to reject false TWA members. Based on the Li $6708 \AA$ equivalent widths, we eliminated TYC 08290845-1, TYC 6604-0118-1, HIP 49530, TYC 6625-1087-1, TYC 7178-1493-1, TYC 7183-1879-1, TYC 7188-0575-1, HIP 47039, HIP 48273, HIP 50796, TYC 7710-2231-1, HIP 52462, HIP 52787, HIP 53486, HIP 57129, HIP 57269, and HIP 59315. HIP 59315 and HIP 57269 have fairly large Li $6708 \AA$ equivalent widths and they may be as young as 30 Myrs, but their Li $6708 \AA$ equivalent widths are too small to be included as TWA members. They are also rejected on additional grounds (see below).

Independent age estimations were obtained for all Hipparcos MF candidates based on their location in a color-magnitude diagram (Fig. 2). We plotted absolute visual magnitude $\left(M_{V}\right)$ versus $(B-V)$ values for all Hipparcos MF candidates where $(B-V)$ values are either ground based measurements (taken from the Hipparcos catalog) or estimated from Tycho $\left(B_{\mathrm{T}}-V_{\mathrm{T}}\right)$ values using a relation given in Bessell (2000). The $M_{V}$ values were calculated from ground-based or Tycho visual magnitudes and Hipparcos parallaxes.

From Fig. 2, we can eliminate most Hipparcos MF candidates except HIP 47039 and HIP 57524. HIP 57524 is an already known TWA member (TWA 19; Zuckerman et al. 2001b). Although the location of HIP 57524 on a color magnitude diagram is not well defined due to a large error, its K7 companion (TWA 19B) confirms its very young age (Fig. 2). Based on a weak Li line and slow rotation, we believe HIP 47039 to be a post-ZAMS star; hence it cannot be a TWA member. HIP 48273 and HIP 57269 are binaries with about equal masses and their absolute magnitudes were adjusted to account for this. The age of HIP 55899 (A0V) cannot be estimated from Fig. 2 because an A0 star evolves very quickly so that in $\sim 10$ Myrs, A0 stars arrive at the zero-age main-squence (ZAMS). Following Lowrance et al. (2000), we plotted all A-type stars with small errors in parallax and $(B-V)$ values from the Hipparcos catalog (Fig. 3). Because of the fast evolution of A-type stars, statistically, young A-type 
Table 1. Spectroscopic data for Makarov and Fabricious TWA candidates.

\begin{tabular}{|c|c|c|c|c|c|c|c|c|c|}
\hline \multirow{2}{*}{$\begin{array}{c}\text { Name } \\
\text { HIP or TYC }\end{array}$} & \multirow{2}{*}{$\begin{array}{c}(B-V)^{\dagger} \\
\text { (error) }\end{array}$} & \multirow{2}{*}{$\begin{array}{c}\text { R.V. } \\
\text { predicted }\end{array}$} & \multicolumn{3}{|c|}{ R.V. measured $\left(\mathrm{km} \mathrm{s}^{-1}\right)$} & \multirow{2}{*}{$\begin{array}{c}E W(\mathrm{H} \alpha) \\
(\AA)\end{array}$} & \multirow{2}{*}{$\begin{array}{c}E W(\mathrm{Li}) \\
(\mathrm{m} \AA)\end{array}$} & \multirow{2}{*}{$\begin{array}{c}v \sin i \\
\left(\mathrm{~km} \mathrm{~s}^{-1}\right)\end{array}$} & \multirow{2}{*}{$\begin{array}{c}\text { TWA } \\
\text { member? }\end{array}$} \\
\hline & & & $\mathrm{SSO}$ & Torres et al. & Others $^{\ddagger}$ & & & & \\
\hline 53911 & $0.64(12)$ & 12.7 & - & $+12.9 \pm 0.3$ & - & -220 & 390 & 4 & TWA 1 \\
\hline 7201-0027-1 & $1.88(37)$ & 10.6 & - & $+11.2 \pm 0.3$ & - & -1.89 & 490 & 13 & TWA 2 \\
\hline 55505 & $1.19(03)$ & 9.1 & - & $+9.2 \pm 0.2$ & - & 0 & 360 & - & TWA 4 \\
\hline $7223-0275-1$ & $1.44(33)$ & 10.0 & $-30.6 \pm 6.6$ & $+6.9: \pm 2.0$ & - & -13.4 & 570 & $36:$ & TWA 5 \\
\hline $7183-1477-1$ & $1.61(26)$ & 16.2 & - & - & - & -4.65 & 560 & - & TWA 6 \\
\hline 7190-2111-1 & $1.31(18)$ & 11.0 & - & - & - & -4.95 & 440 & - & TWA 7 \\
\hline 57589 & $1.66(40)^{G}$ & 12.6 & - & $+10.2 \pm 0.4$ & - & -5.01 & 480 & - & TWA 9 \\
\hline 61498 & $0.00(01)^{G}$ & 10.5 & - & - & $+9.4 \pm 2.3$ & - & - & - & TWA 11 \\
\hline 57524 & $0.60(02)$ & 20.0 & $+11.5 \pm 3.8$ & - & - & 0.57 & 189 & 24 & TWA 19 \\
\hline 46535 & $0.50(01)^{G}$ & 16.0 & $+18.0 \pm 7.0$ & - & $+22.1 \pm 5.0$ & 1.43 & 63 & 37 & no \\
\hline 47039 & $0.42(01)^{G}$ & 16.9 & $+10.9 \pm 1.3$ & - & $+12.2 \pm 0.4$ & 1.63 & $<10$ & 8 & no \\
\hline 48273 & $0.48(01)^{G}$ & 10.7 & $-31.4 \pm 2.9$ & $+16.2 \pm 0.1$ & $+17.0^{\S}$ & 2.0 & $21 \& 22$ & $9 \& 14$ & no \\
\hline 0829-0845-1 & $0.69(05)$ & 20.4 & $+9.5 \pm 6.5$ & - & - & 0.85 & $<10$ & 30 & no \\
\hline 6604-0118-1 & $1.07(06)$ & 16.5 & $-19,+54$ & $+27.0 \pm 0.3$ & - & -0.5 & 93 & 20 & no \\
\hline 49530 & $0.94(01)^{G}$ & 20.7 & $+22.5 \pm 1.1$ & - & $+16.7 \pm 2.0$ & 1.17 & 34 & 2 & no \\
\hline $6625-1087-1$ & $0.87(13)$ & 21.3 & $+16.1 \pm 1.5$ & - & - & 0.28 & 141 & 10 & no \\
\hline 7178-1493-1 & $0.73(18)$ & 15.2 & $+48.0 \pm 3.0$ & - & - & 0.17 & $<10$ & 15 & no \\
\hline 7183-1879-1 & $0.96(20)$ & 18.9 & $+11.0 \pm 1.6$ & - & - & 0.74 & 79 & 11 & no \\
\hline 7188-0575-1 & $1.11(05)$ & 12.2 & $+42.9 \pm 2.1$ & - & - & -0.28 & 50 & 25 & no \\
\hline 50796 & $1.19(02)^{G}$ & 13.1 & $+22.4 \pm 0.9$ & $+13.1 \pm 1.0$ & - & 0.20 & $<10$ & 8 & no \\
\hline $7710-2231-1$ & $1.07(03)$ & 22.5 & $-12.2 \pm 1.0$ & - & - & 0.63 & $<14$ & 7 & no \\
\hline 52462 & $0.87(01)^{G}$ & 9.0 & $+22.7 \pm 0.5$ & - & - & 0.78 & 150 & 1 & no \\
\hline 52787 & $0.82(02)$ & 9.9 & $+24.0 \pm 0.6$ & - & - & 0.82 & 120 & 2 & no \\
\hline 53486 & $0.91(02)^{G}$ & 3.7 & $+4.3 \pm 1.0$ & $+5.5 \pm 0.3$ & - & 0.88 & $<10$ & 1 & no \\
\hline 55899 & $0.07(02)$ & 21.8 & - & - & - & 2.0 & - & 210 & no? \\
\hline 57129 & $0.54(03)$ & 12.9 & $+15 \pm 20$ & - & $+25.6 \pm 10$ & 1.56 & $<10$ & 190 & no \\
\hline 57269 & $0.91(01)^{G}$ & 8.5 & - & - & $+15.9^{\star}$ & - & 196 & 20 & no \\
\hline 59315 & $0.71(01)^{G}$ & 6.6 & $+15.9 \pm 0.6$ & - & - & 0.83 & 151 & 5 & no \\
\hline 7760-0835-1 & $0.51(03)$ & 19.4 & $+10.0 \pm 2.6$ & - & - & 0.83 & 161 & 12 & yes? \\
\hline 8238-1462-1 & $0.77(04)$ & 16.8 & $+12.0 \pm 3.0$ & - & - & 0.36 & 294 & 18 & yes? \\
\hline 8234-2856-1 & $0.81(05)$ & 20.1 & $+13.2 \pm 2.4$ & - & - & -0.49 & 342 & 16 & yes? \\
\hline
\end{tabular}

${ }^{\dagger}$ Estimated from Tycho $\left(B_{\mathrm{T}}-V_{\mathrm{T}}\right)$ using a relation given in Bessell (2000).

Stars with superscript " $G$ ", $(B-V)$ colors are from ground photometry adapted from Hipparcos Input Catalog.

$¥$ Radial velocities from Barbier-Brossat \& Figon (2000) unless noted otherwise.

$\S$ Radial velocity from Mayor \& Mazeh (1987).

$\star$ Radial velocity from Anders et al. (1991).

stars have to be located at the bottom of the A-stars' distribution (ZAMS), as seen in Fig. 3 for the three young A-type stars (HR 4796A = TWA 11, HD 141569, and $\beta$ Pictoris). HIP 55899 is not located on the ZAMS, thus it is unlikely to be as young as the other three stars. From Figs. 1-3, we eliminated all candidates except HIP 57524 (TWA 19) and three Tycho stars (7760-0835-1, 8238-14621 , and 8234-2856-1).

From their Li $6708 \AA$ strengths, positions in the sky (RA $\sim 12 \mathrm{~h}$, DEC $\left.\sim-45^{\circ}\right)$, and proper motions $\left(\mu_{\alpha} \sim\right.$ $-34 \mathrm{mas} / \mathrm{yr}$ and $\mu_{\delta} \sim-12 \mathrm{mas} / \mathrm{yr}$ ), the three Tycho stars must be members of the Lower Centaurus Crux (LCC) association. Assuming they are $\sim 10 \mathrm{Myr}$ old stars, we can photometrically estimate their distance $(\sim 130 \mathrm{pc})$. Previously known TWA members are likely $\leq 100 \mathrm{pc}$ from Earth. Now having eliminated all the distant TWA MF candidates (other than the three Tycho stars), we cannot confirm or reject a possible connection between TWA and the LCC. Additional data, especially identification of less massive members of the Sco-Cen complex, are required to further investigate the possibility of such a connection. Currently known members of the complex are mostly 


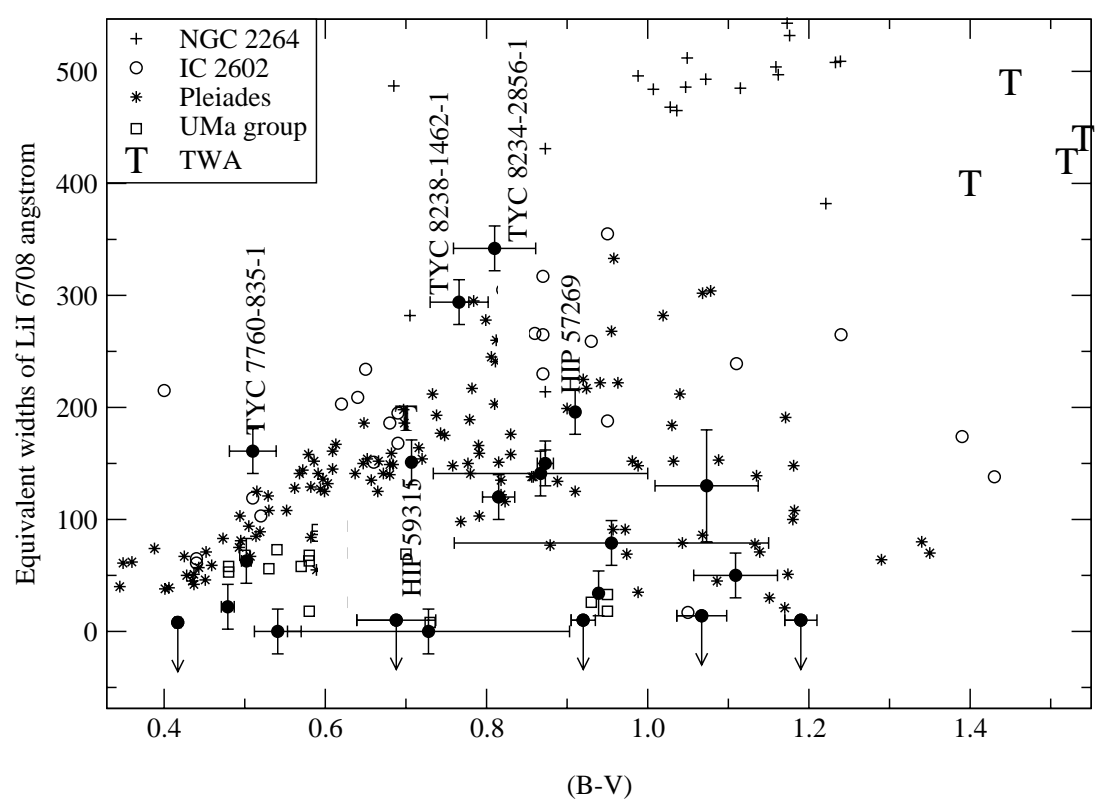

Fig. 1. Equivalent widths of $\operatorname{Li} 6708 \AA$ as a function of $(B-V)$. Displayed equivalent widths are not corrected for the possible contamination by FeI $6707.44 \AA$ and measurement uncertainty of $E W(\mathrm{Li})$ is about $20 \mathrm{~m} \AA$. Plus signs indicate NGC 2264 stars (<10 Myrs), open circles denote IC 2602 stars (10-30 Myrs), star symbols represent Pleiades stars ( 125 Myrs), and open squares are Ursa Majoris moving group stars ( 300 Myrs). Previously known TWA members are plotted as the symbol "T" and solid circles indicate MF candidates.

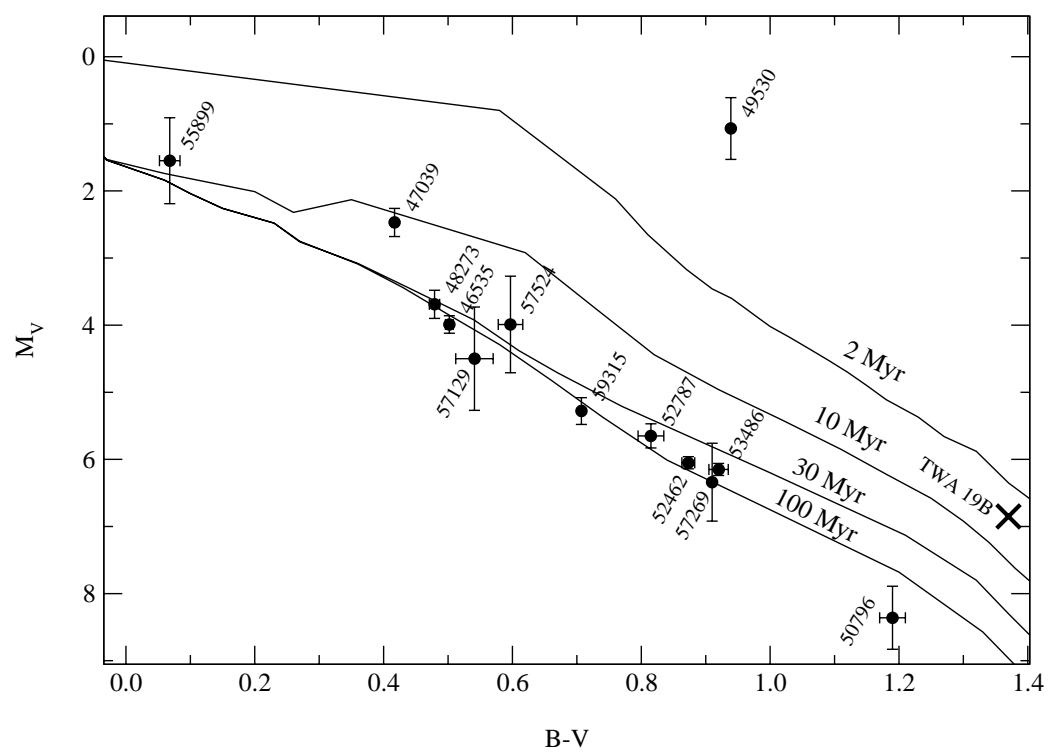

Fig. 2. Color magnitude diagram of Hipparcos MF candidates along with theoretical isochrones from Siess et al. (2000) $Z=0.02$ model. HIP 48273 and HIP 57269 are known binaries with almost equal masses and the plotted points were corrected for this; that is, $M_{v}$ corresponds to that of one member. HIP 49530 is a giant star (K1 III) and HIP 57129 is a W UMa type contact binary (Sistero \& Sistero 1973), thus they cannot be members of TWA. TWA 19B (a K7 companion of HIP 57524) is plotted by using $R \& I$ photometric data $(R=11.06$ and $I=10.21)$ from Zuckerman et al. (2001b) and distance of the primary $(104 \mathrm{pc})$.

earlier than F/G-type stars (de Zeeuw et al. 1999). If TWA is not a part of the LCC/Sco-Cen complex, based on the proximity of TWA 19 to LCC in space, it is also possible that TWA 19 belongs to LCC rather than to TWA itself.

In summary, among 23 Makarov \& Fabricius (2001) TWA candidates, we verify only three possibly new TWA members (TYC 7760-0835-1, TYC 8238-1462-1, and
TYC 8234-2856-1). If TWA is not a part of a larger structure (the LCC/Sco-Cen complex), then these three Tycho stars may not be related to TWA. Therefore, MF's claims of an isotropic expansion of TWA and TWA being a part of the "Gould-disk" have to be re-evaluated based on our new results. It is clearly shown that in identifying new members of nearby diffuse stellar associations or groups, 


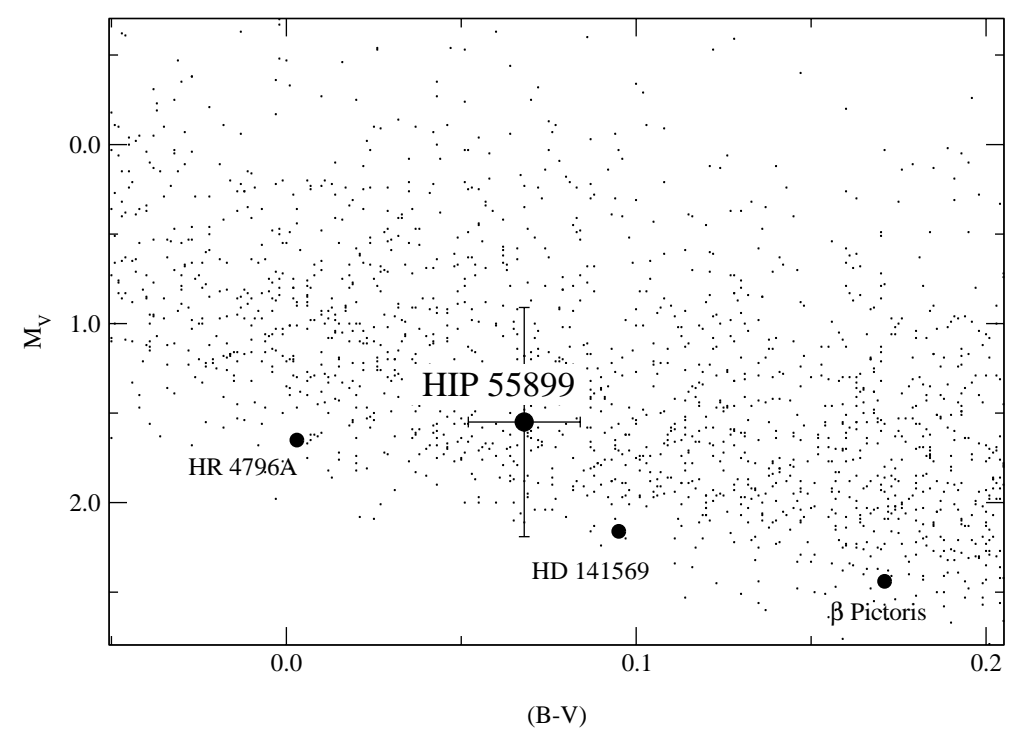

Fig. 3. A-type stars color magnitude diagram. Dots represent Hipparcos A-type stars with small parallax error $(<10 \%)$ and small $(B-V)$ error $(<0.05 \mathrm{mag})$. HIP 55899 is plotted with error bars. Three well known young ( $\leq 12$ Myrs) A-type stars are displayed as solid circles.

one cannot rely solely on kinematic properties of candidates. When photometric - putting the candidates in a color magnitude diagram - and spectroscopic - Li $6708 \AA$ and $\mathrm{H} \alpha$ - data are combined with kinematic information, much more convincing membership can be achieved as seen in Zuckerman et al. (2001a).

Acknowledgements. We thank Dr. van den Ancker for helpful remarks as the referee. This research was supported in part by the UCLA Astrobiology Institute and by a NASA Origins grant to UCLA.

\section{References}

Anders, G. J., Coates, D. W., Thompson, K., \& Innis, J. L. 1991, MNRAS, 252, 408

Barbier-Brossat, M., \& Figon, P. 2000, A\&AS, 142, 217

Bessell, M. S. 2000, PASP, 112, 961

de Zeeuw, P. T., Hoogerwerf, R., de Bruijne, J. H. J., Brown, A. G. A., \& Blaauw, A. 1999, AJ, 117, 354

Jayawardhana, R., \& Greene, T. (ed.) 2001, Young Stars Near Earth: Progress and Prospects, ASP Conf. Ser. 244
Lowrance, P. J., McCarthy, C., \& Becklin, E. E., et al. 1999, ApJL, 512, L69

Lowrance, P. J., Schneider, G., Kirkpatrick, J. D., et al. 2000, ApJ, 541, 390

Makarov, V. V., \& Fabricius, C. 2001, A\&A, 368, 866

Mayor, M., \& Mazeh, T. 1987, A\&A, 171, 157

Patience, J., Ghez, A. M., Reid, I. N., Weinberger, A. J., \& Matthews, K. 1998, AJ, 115, 1972

Siess, L., Dufour, E., \& Forestini, M. 2000, A\&A, 358, 593

Sistero, R. F., \& Sistero, M. E. C. 1973, AJ, 78, 413

Strassmeier, K. G., Fekel, F. C., Bopp, B. W., Dempsey, R. C., \& Henry, G. W. 1990, ApJS, 72, 191

Torres, G., Neuhaeuser, R., \& Latham, D. W. 2001, in Young Stars Near Earth: Progress and Prospects, ed. R. Jayawardhana, \& T. Greene, ASP Conf. Ser., 244, 283

van den Ancker, M. E., Pérez, M. R., \& de Winter, D. 2001, in Young Stars Near Earth: Progress and Prospects, ed. R. Jayawardhana, \& T. Greene, ASP Conf. Ser., 244, 69

Zuckerman, B., Song, I., Bessell, M. S., \& Webb, R. A. 2001a, ApJ, 562, L87

Zuckerman, B., Webb, R. A., Schwartz, M., \& Becklin, E. E. 2001b, ApJ, 549, L233 